\title{
Morphological Analyses of Sex Differences and Age-Related Changes in C3H Mouse Midbrain
}

\author{
Takashi TANIDA ${ }^{1)}$, Katsuhiko WARITA ${ }^{1,2)}$, Tomoko MITSUHASHI ${ }^{1)}$, Kana ISHIHARA ${ }^{1)}$, Toshifumi YOKOYAMA ${ }^{1)}$, \\ Hiroshi KITAGAWA $^{1)}$ and Nobuhiko HOSHI ${ }^{1) *}$ \\ ${ }^{1)}$ Laboratory of Functional Morphology, Department of Animal Science, Graduate School of Agricultural Science, Kobe University, Kobe \\ 657-8501 and ${ }^{2)}$ Department of Anatomy and Neurobiology, Faculty of Medicine, Kagawa University, Kagawa 761-0793, Japan
}

(Received 22 June 2008/Accepted 8 September 2008)

ABSTRACT. While it is commonly hypothesized that sexual differentiation in the mammalian brain is initiated mainly by gonadal sex steroids, recent evidence has suggested that dopaminergic (DA) neurons within the rodent midbrain have sex differences independent of gonadal secretions. More recently, it has been reported that Sry (the sex-determining region of the Y chromosome) is directly involved in this difference. The possibility of sexual dimorphism in the mouse midbrain needs to be elucidated. In the present study, the midbrain of $\mathrm{C} 3 \mathrm{H}$ mice, which is little understood, was investigated histologically and immuno-histoplanimetrically to reveal sexual and developmental differences. The female ventral tegmental area appeared to contain higher immunoreactivity to tyrosine hydroxylase (TH) than that of males at 11 weeks of age, whereas general histological differences between the sexes were not clearly found. The THimmunoreactive (TH-ir) neurons within the A8, A9, and A10 mesencephalic areas were examined separately. There was the sex difference in the time period when TH-ir cell numbers significantly increased, indicating that the growth rate of midbrain DA nuclei also differs and that the midbrain DA system may trace different processes of sexual maturation between the sexes. These differences between female and male may reflect the direct regulation by Sry or the multiple effects of both Sry and sex steroids. Further experiments are needed to determine which factor forms this difference in the growth pattern in the numbers of TH-ir neurons.

KEY WORDS: age-related change, dopaminergic (DA) neuron, midbrain, sex difference, tyrosine hydroxylase (TH).

J. Vet. Med. Sci. 71(7): 855-863, 2009

Sex differentiation of the mammalian brain is influenced mainly by perinatal testicular steroid hormones [25]. In males, androgen (or its metabolite estrogen) initiates a program of male-specific development in many brain regions. In females, on the other hand, neural development traces female-specific processes caused by a low level of gonadal steroid hormone. These mechanisms result in morphological sexual dimorphism, which controls sexually dimorphic behaviors and functions [16]. Sex steroid hormones are associated with the constitution of several sexual dimorphisms via estrogen receptor (ER) [22]. Moreover, the sexual differentiation of dopaminergic (DA) neurons within the preoptic region of the diencephalon depends on ER [32].

However, other sex differentiation processes have been found to depend on the sex chromosomal gene directly rather than on gonadal steroid hormones in the midbrain. The cell cultures dissociated from female rat mesencephalon on embryonic day 14 (ED 14; before exposure to the gonadal steroid hormone shower) contain higher numbers of DA neurons than male cultures, and hormonal treatment does not erase this differentiation [4]. The sex-determining region of the Y chromosome (Sry) is transcribed in the hypothalamus, mesencephalon, and testis in adult (3-month-old) male but not in adult female mice [23]. Sry transcripts were also found in the male

\footnotetext{
* Correspondence to: Hoshi, N., Graduate School of Agricultural Science, Kobe University, 1-1, Rokkodai, Nada-ku, Kobe 6578501, Japan.

e-mail: nobhoshi@kobe-u.ac.jp
}

diencephalon, midbrain, and cortex in the postnatal day (d) period between 14 and 90 [26]. These reports indicate that Sry has a crucial role in regulating the sex differentiation of the mammalian central nervous system (CNS). The sex difference in the mesencephalon is also found in Parkinson's disease (PD), a progressive neurodegenerative disease characterized by the selective loss of DA neurons from the substantia nigra ( $\mathrm{SN}$ ) and the concomitant appearance of motor disturbances including tremors, rigidity, and slowness of movement (akinesia). Many studies have indicated that the prevalence of PD is 1.36-3.7 times higher in men than in women $[2,5,6,14]$. Dewing et al. (2003) detected at least seven murine genes that show differential expression between female and male mouse developing brains at ED 10.5 before any gonadal hormone influence [13]. For these reasons, the midbrain likely has sex differences of some kind. In fact, sexual dimorphism was found in the tyrosine hydroxylase (TH) activity of SN and in the exploratory behavior of both BALB/cJ and CBA/ $\mathrm{J}$ inbred mouse strains [37].

Despite these many demonstrations of sex differences in the midbrain, research also indicates that there is no sex difference within mesencephalic DA nuclei. Lieb et al. (1996) investigated the numbers of DA neurons in the mesencephalon of male and female $\mathrm{CBA} / \mathrm{J}$ mice between ED 13 and $90 \mathrm{~d}$, but they could not find statistically significant sex differences in the numbers of DA neurons at any time point [24]. In addition, there is a genetic control of the neuron number (including midbrain DA neurons) within inbred strains of mice $[1,39]$. Presently, however, we still 
lack detailed information about sex and/or genetic strain differences within the midbrain.

There are other reasons why mesencephalic DA neurons are of interest: midbrain DA nuclei, which play crucial roles in cognition, emotion, and behavior, are also significant in clinical medicine because their functional disorder causes various neurodegenerative diseases, such as schizophrenia [27], PD [18], substance abuse [20], and disturbances in the control of motor activity and learning [3], attention [38], and other behaviors including attention-deficit hyperactivity disorder [15].

Mesencephalic DA nuclei are distributed in the A8, A9, and $\mathrm{A} 10$ areas [11]. A8 contains the retrorubral field (RRF); A9 consists of the $\mathrm{SN}$ pars compacta $(\mathrm{SNc}), \mathrm{SN}$ pars reticulata $(\mathrm{SNr})$, and $\mathrm{SN}$ pars lateralis $(\mathrm{SNl})$; and $\mathrm{A} 10$ is composed of the ventral tegmental area (VTA), parabrachial pigmented nucleus (PBP), paranigral nucleus (PN), and central linear nucleus (CLi). Neurons in the A9 area have projective fibers toward the striatum and form the nigrostriatal dopaminergic system, which promotes the extrapyramidal function. The DA neurons within A10 project to the subcortical and cortical limbic areas as well as to the prefrontal cortex, while A8 neurons provide dopaminergic input to both the striatum and the limbic areas. A DA neuronal deficit within the A10 area contributes to drug addiction and is thought to be a point of action by schizophrenia-curative drugs [30].

In the present study, male and female $\mathrm{C} 3 \mathrm{H}$ mice, whose midbrain is poorly understood, were examined at $3 \mathrm{~d}$ (neonatal period), 21d (pre-puberty), and 11 week (wk) of age (post-puberty) to elucidate the sex differences and agerelated changes within the midbrain of this inbred strain during sexual maturation.

\section{MATERIALS AND METHODS}

Animals: Female and male mice of the $\mathrm{C} 3 \mathrm{H} / \mathrm{He}$ strain were purchased from Japan SLC, Inc. (Shizuoka, Japan) for breeding stock. The animals used for the experiments were bred in a laboratory at the Department of Animal Science at Kobe University, under a $12 \mathrm{hr}$ light/dark cycle at $21-24^{\circ} \mathrm{C}$ and 40-60\% humidity. A laboratory diet (Lab MR Stock; Nosan Corp., Yokohama, Japan) and filtered water were available ad libitum. The experiments were conducted according to the Guidelines for the Care and Use of Laboratory Animals of Kobe University. Nineteen mice were divided into three age groups-3d $(n=5), 21 d(n=8)$, and $11 \mathrm{wk}(\mathrm{n}=6)$--in each sex.

Tissue preparation: We gave careful attention to each experimental treatment in order to maintain the equality of all brain samples. At the time of necropsy, mice were deeply anesthetized with diethyl ether and were subcutaneously injected with heparin ( $1 \mathrm{U} / \mathrm{g}$ body weight) to avoid blood coagulation. After $10 \mathrm{~min}$, the mice were transcardially perfused with $0.1 \mathrm{M}$ ice-cold phosphate buffer (PB), $\mathrm{pH} 7.4$, including 3.4 mM EDTA, followed by perfusion with ice-cold $4 \%$ paraformaldehyde in $\mathrm{PB}$. The brains were post-fixed with the same fixative overnight at $4^{\circ} \mathrm{C}$, dehydrated through a graded series of ethanol followed by xylene, and embedded in paraffin. Coronal serial

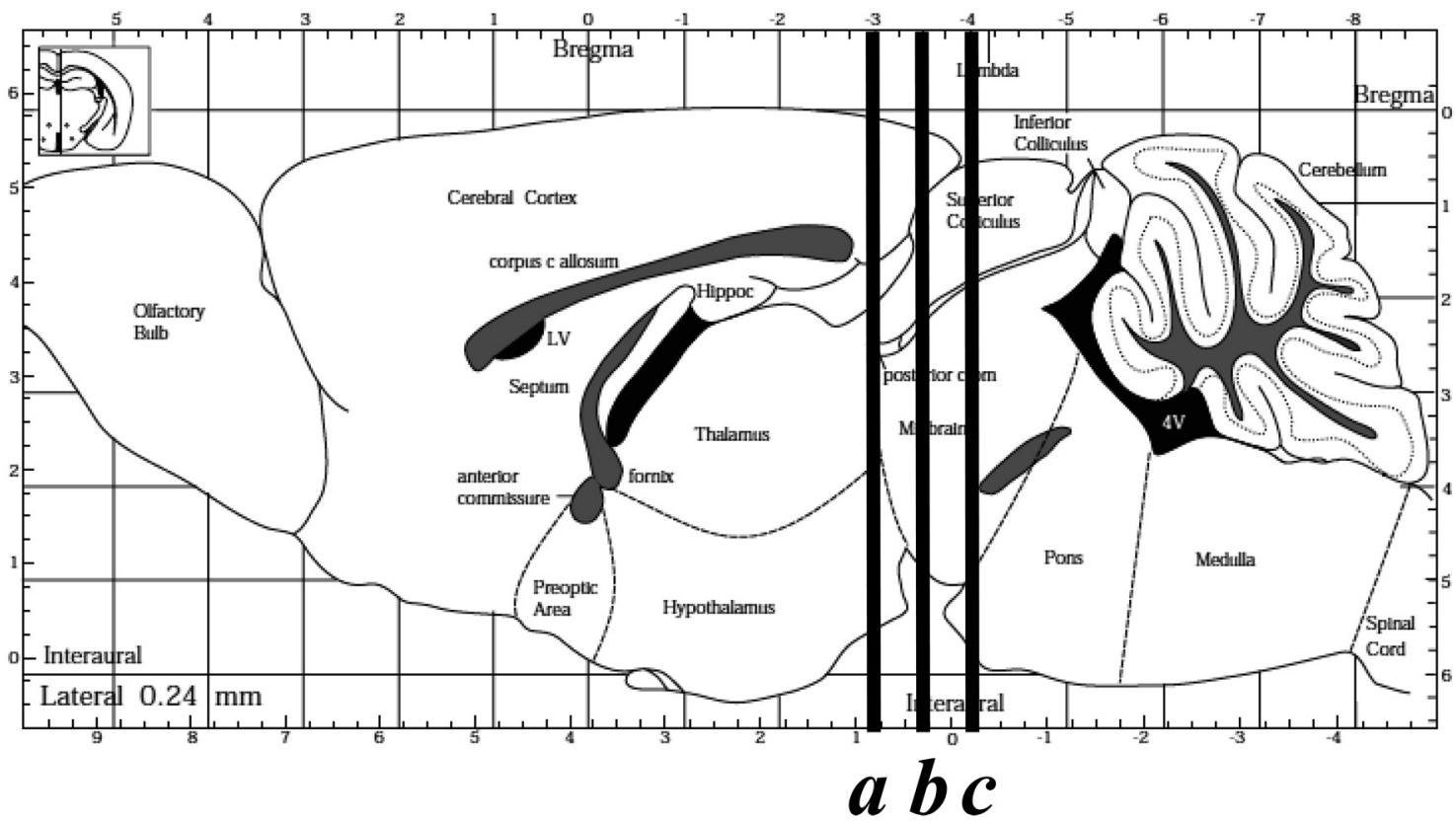

Fig. 1. Sagittal section of the mouse brain referred from The Mouse Brain in Stereotaxic Coordinates [31]. The transverse lines of $a, b$, and $c$ correspond to the rostral, intermediate, and caudal parts of the midbrain, respectively. The lines show brief coordinates of the following figures. Line $a$ corresponds to coronal photomicrographs of Fig. 2, Fig. 3A-C, and Fig. 4A, D, G, J, M, P. Line $b$ corresponds to coronal photomicrographs of Fig. 3D-F and Fig. 4B, E, H, K, N, Q. Line $c$ corresponds to coronal photomicrographs of Fig. 3G-L and Fig. 4C, F, I, L, O, R. 
sections of $10 \mu \mathrm{m}$ in thickness were then cut, and each section was mounted on a glass slide precoated with silane (Matsunami, Osaka, Japan).

Cresyl fast violet staining: A series of sections was made by collection at $80-100 \mu \mathrm{m}$ intervals. After deparaffinization and rehydration, the sections were stained with $0.1 \%$ cresyl fast violet solution, which visualizes Nissl's substance for $10 \mathrm{~min}$. They were then placed in distilled water (DW) and a graded series of ethanol for appropriate bleaching of dye, dehydrated with ethanol, cleared by xylene, and coverslipped with Eukitt. Each section was morphologically categorized by using a guide [31] (Fig. 1).

Immunohistochemistry: The immunoreactivity of TH was applied to visualize DA neurons. Deparaffinized tissue sections were analyzed using a Histofine Mouse Stain Kit (Nichirei Bioscience, Tokyo, Japan) for monoclonal antibody in the mouse tissues following the manufacturer's instructions. After immersion in $100 \%$ methanol including $3 \% \mathrm{H}_{2} \mathrm{O}_{2}$ for $30 \mathrm{~min}$ at room temperature (RT) to quench endogenous peroxidase activity, the sections were incubated with the primary antibody against TH (MAB-318; Chemicon International, Temecula, CA, U.S.A.) diluted $1: 1,200$ in phosphate-buffered saline at $4{ }^{\circ} \mathrm{C}$ overnight. Immunoreactivity was then detected by incubation with 3,3'-diaminobenzidine solution (Dako, Glostrup, Denmark) containing $0.03 \% \mathrm{H}_{2} \mathrm{O}_{2}$. The sections were counterstained lightly with $0.1 \%$ cresyl fast violet solution for $1 \mathrm{~min}$. Finally, they were placed in DW and a graded series of ethanol, dehydrated with absolute ethanol, cleared by xylene, and coverslipped with Eukitt. The distribution of each DA nucleus (A8, A9, and A10) within the midbrain was classified morphologically (Fig. 1). To compare the staining intensity across different sections, each semi-serial section was captured by a digital still camera (DS-L1; Nikon, Tokyo, Japan) connected to a light microscope. The background staining intensity was manually adjusted to the regions lacking $\mathrm{TH}$ and $\mathrm{Nissl}$ staining in each microphotograph. Then the immunoexpression within the perikarya of each DA nucleus (A8, A9, and A10) was evaluated (data not shown).

Histoplanimetrical analysis: The coronal semi-serial sections stained immunohistochemically were observed from the caudal level of the diencephalon to the rostral level of the pons (Fig. 1) at magnifications of $\times 200$ using an Olympus light microscope. The first sampling tissue section for counting was taken when the most rostral THimmunoreactive (TH-ir) cells were observed in the midbrain unilaterally (right side), and all the following sections were taken at intervals of $80-100 \mu \mathrm{m}$ from the previous one. Then only neurons containing perikarya clearly stained for $\mathrm{TH}$ within the right side of the A8, A9, and A10 areas were manually and separately counted through the entire midbrain, and the total $(\mathrm{A} 8+\mathrm{A} 9+\mathrm{A} 10)$ number of neurons was calculated.

Statistical analysis: Unpaired $t$-test (StatView version 5.0; SAS Institute, Cary, NC, U.S.A.) was applied to determine the statistical differences in the TH-ir cell numbers by sex and age. Analyses were considered statistically significant at $P<0.05$.

\section{RESULTS}

The age-related changes found in both cresyl fast violet and immunostained sections are shown in Figs. 2 and 3. In general, all of the neurons we investigated were multipolar, which are most typical in the CNS, and the perikarya and cellular nuclei increased slightly in size as the mice developed. The volume of nuclei also increased concomitantly with brain development. In the cresyl fast violet-stained sections, the nuclei composed of round or ovoid cells with short dendrites were arcuate hypothalamic nuclei (Arc), interpeduncular nuclei (IP), PN, CLi, and pontine nuclei $(\mathrm{Pn})$. On the other hand, nuclei whose perikarya (with comparatively long dendrites) appeared stellate or pyramidal were SNc, SNl, RRF, and VTA. Extremely large cells were distributed within oculomotor nuclei $(3 \mathrm{~N})$ and red nuclei $(\mathrm{Rn})$ in both sexes. These findings were common between the sexes. The findings observed in TH-immunostained sections of both sexes were as follows: $\mathrm{TH}$-ir cells contained deeply stained perikarya and round unstained nuclei. SNc seemed to consist of the largest TH-ir cells in the midbrain DA nuclei (Fig. 3). CLi and PN appeared to contain comparatively small $\mathrm{TH}$-ir cells, and their immunostained fibers were comparatively short (Fig. 3D-I). The regions in which TH-ir cells projected comparatively long fibers were observed in SNc,
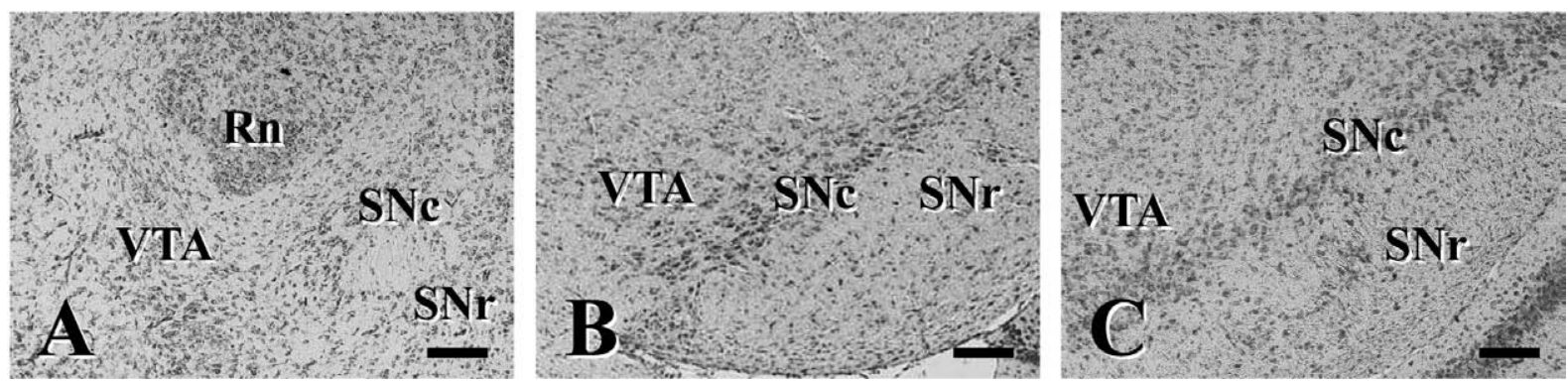

Fig. 2. Age-related changes of rostral midbrain in cresyl fast violet-stained sections corresponding to the transverse line $a$ in Fig. 1 . Coronal photomicrographs show changes at $3 \mathrm{~d}(\mathrm{~A}), 21 \mathrm{~d}(\mathrm{~B})$, and $11 \mathrm{wk}(\mathrm{C})$. The size of perikarya and cellular nuclei constituting each neuronal nucleus increases slightly, as do the contents of neuronal nuclei in parallel with brain development. Bar=100 $\mu \mathrm{m}$. 

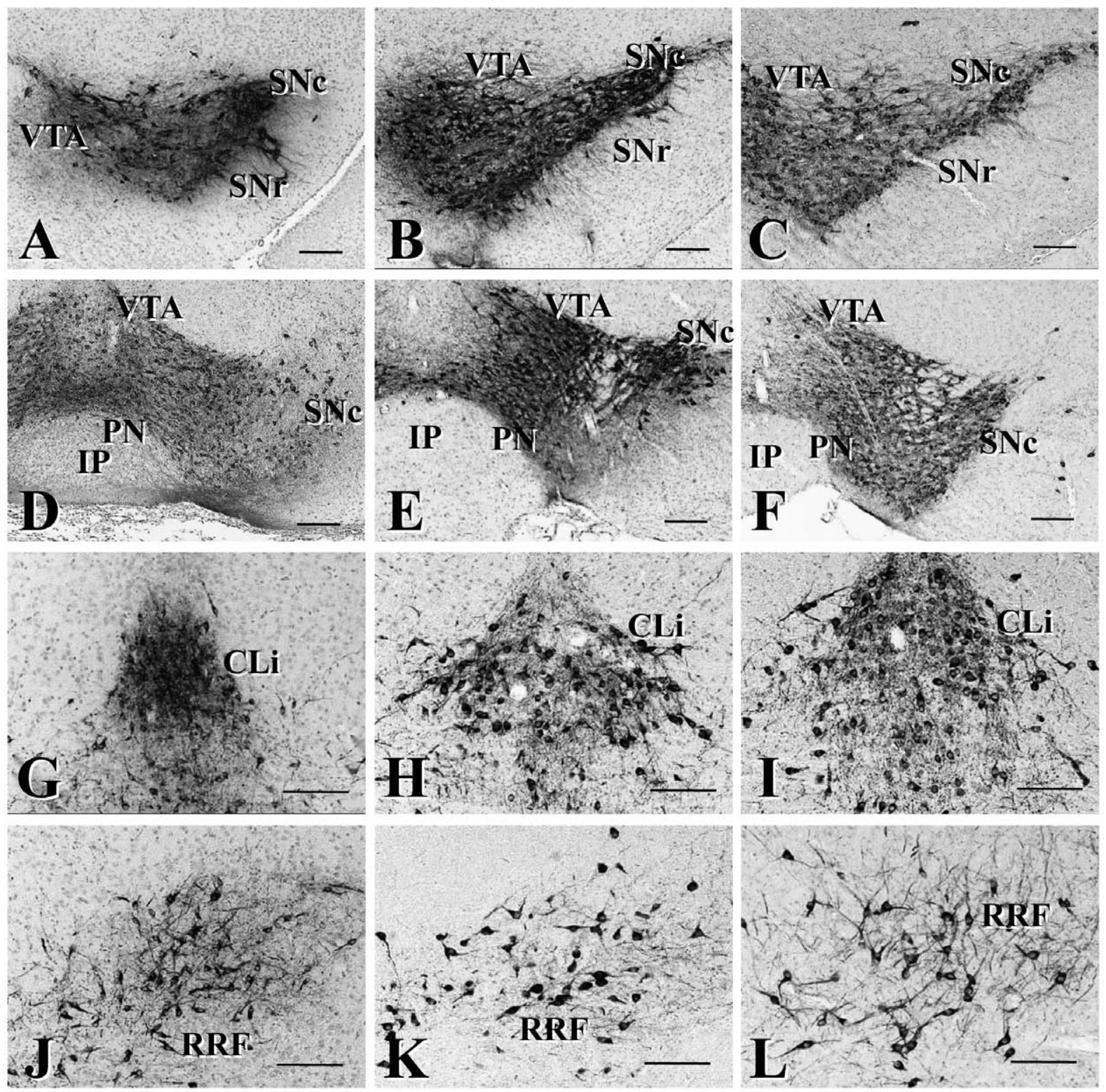

Fig. 3. Age-related changes of DA nuclei within rostral (A-C), middle (D-F), and caudal (G-L) levels of midbrain containing TH-ir cells at the age of $3 \mathrm{~d}(\mathrm{~A}, \mathrm{D}, \mathrm{G}$, and J), 21d (B, E, H, and K), and $11 \mathrm{wk}(\mathrm{C}, \mathrm{F}, \mathrm{I}$, and L). TH-ir perikarya become slight larger, and the volumes of TH-ir nuclei increase in parallel with brain development. Bar=100 $\mu \mathrm{m}$.

SNl, and RRF (Fig. 3). The cell bodies were pyramidal or spindled within SNc, SNl, and VTA (Fig. 3A-F) but were round and ovoid within CLi and PN (Fig. 3D-I).

The immunohistochemical comparison between the sexes is shown in Fig. 4. Although the greatest individual differences were observed at $3 \mathrm{~d}$, the differences did not appear to depend on sex (Fig. 4A-F). Nor was any sexual dimorphism more apparent than individual differences in $21 \mathrm{~d}$ mice (Fig. 4G-L). In the $11 \mathrm{wk}$ mouse midbrain, female VTA appeared to contain higher immunoreactivity of $\mathrm{TH}$ than male VTA (Fig. 4M-R). This sex difference seemed to contribute to the significant increases in A10 TH-ir cells at

\section{$3 \mathrm{~d}, 21 \mathrm{~d}$, and $11 \mathrm{wk}$}

The numbers of TH-ir neurons within midbrain DA nuclei (A8, A9, A10, and total) and the statistical outline of this study are summarized in Figs. 5 and 6, respectively. There were no significant sex differences in the number of TH-ir neurons when they were tested between age-matched groups. However, when the increment in TH-ir cell numbers was examined between 3 and $21 \mathrm{~d}$ and between $21 \mathrm{~d}$ and $11 \mathrm{wk}$, there was the sex difference in the time period when the numbers significantly increased (Figs. 5 and 6); the numbers of female A9, A10, and total TH-ir neurons increased significantly between 3 and 21d (Figs. 5B, C, D, 


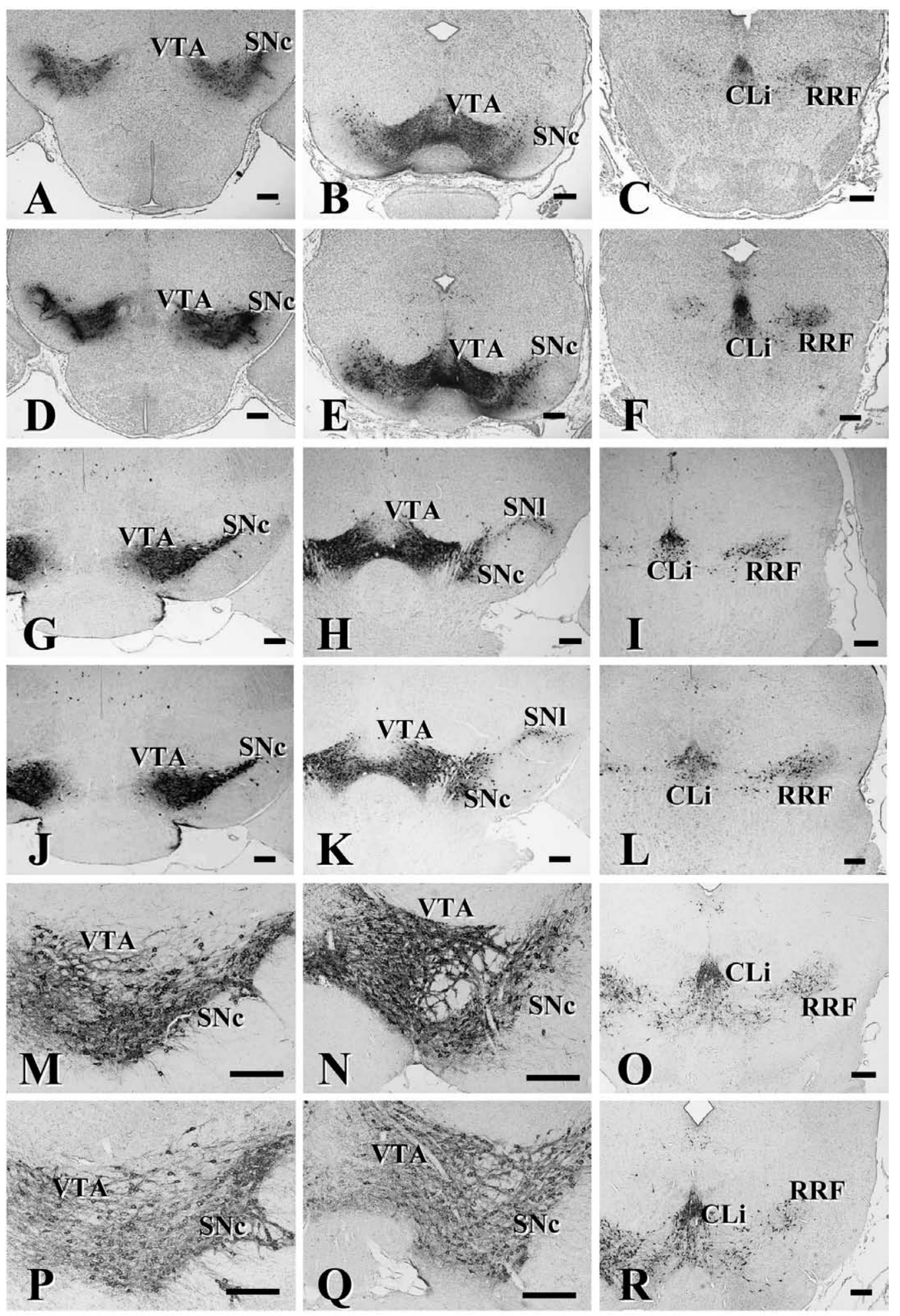

Fig. 4. Immunohistochemical comparison between female (A-C, G-I, and M-O) and male (D-F, J-L, and P-R) mice at 3d (A-F), 21d (G-L), and 11wk (M-R). Coronal figures show rostral (A, D, G, J, M, and P), middle (B, E, H, K, $\mathrm{N}$, and $\mathrm{Q})$, and caudal $(\mathrm{C}, \mathrm{F}, \mathrm{I}, \mathrm{L}, \mathrm{O}$, and R) levels of midbrain. Although individual differences are observed within the midbrain of $3 \mathrm{~d}$ mice, sex differences appear to be independent of the sex. Bar $=200 \mu \mathrm{m}$. 

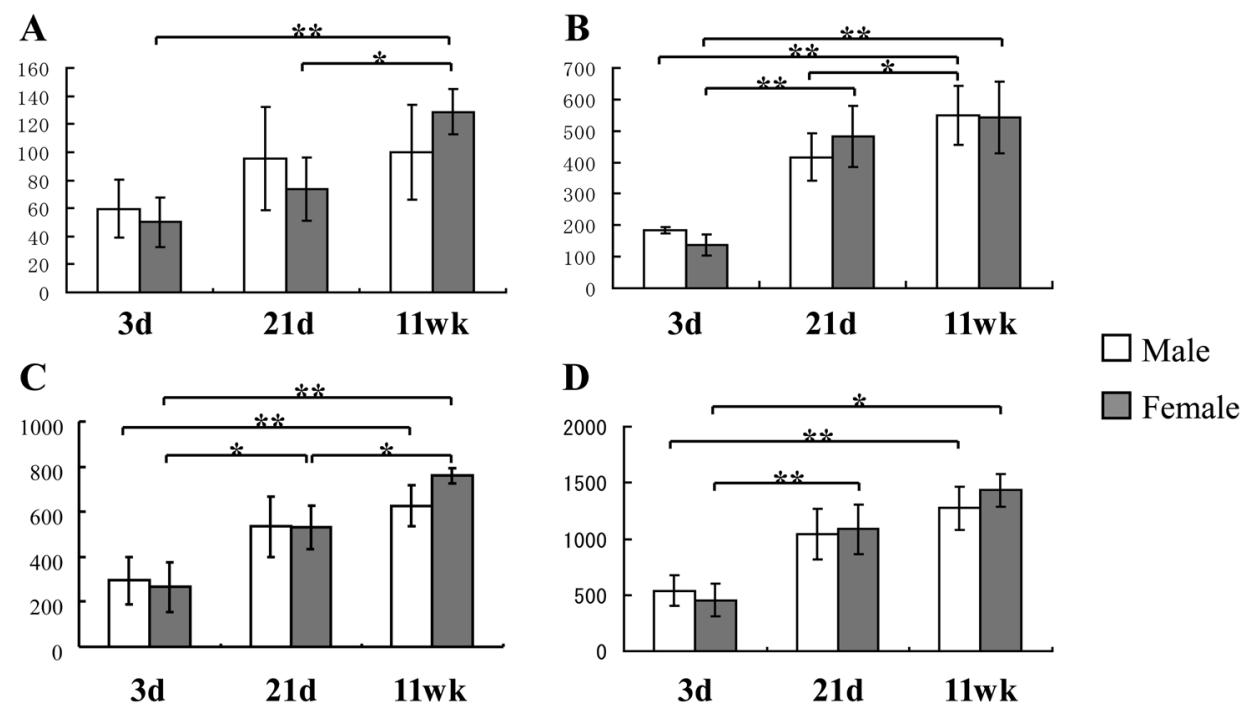

Fig. 5. TH-ir neurons counted within semi-serial sections of A8 (A), A9 (B), A10 (C), and total (D) in female (solid column) and male (open column) 3d, 21d, and $11 \mathrm{wk}$ mice. Significant differences are not detected in the number of TH-ir neurons between the sexes when we tested between age-matched groups, while the time period in which the number significantly increased differs between females and males. In a comparison between $3 \mathrm{~d}$ and $11 \mathrm{wk}$ mice, all nuclei except for male A8 area significantly increase $\mathrm{TH}$-ir neuron numbers. The A9 and A10 areas of both sexes and the female A8 area show a significant increase of $P<0.01$. Values are means \pm SD. *: $P<0.05, * *: P<0.01$.

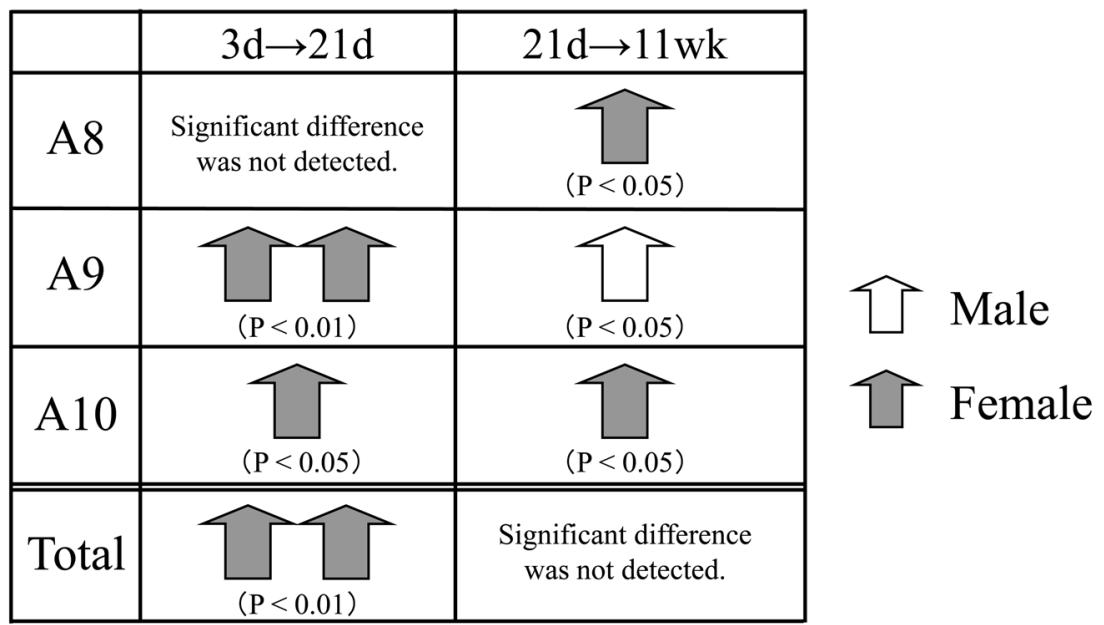

Fig. 6. The statistical outline of this study. The significant increase of the numbers of TH-ir neurons is detected and its increment patterns differ between sexes. It is notable that females show significant increases within all nuclei while males do so only within the A9 area.

and 6); and the numbers of female A8, A10, and male A9 TH-ir neurons increased significantly between $21 \mathrm{~d}$ and 11 wk (Figs. 5A, B, C and 6). In the A8 area, the numbers of $\mathrm{TH}$-ir neurons increased significantly from $21 \mathrm{~d}$ to $11 \mathrm{wk}$ in females only, while neither sex showed significant increases in the numbers of TH-ir neurons between 3 and 21d (Figs. $5 \mathrm{~A}$ and 6 ). The numbers of $\mathrm{TH}$-ir neurons within the $\mathrm{A} 9$ area reached to adult level before $21 \mathrm{~d}$ in female mice, whereas in males the numbers did between $21 \mathrm{~d}$ and $11 \mathrm{wk}$. Among females, the numbers of A10 TH-ir neurons increased significantly from 3 to $21 \mathrm{~d}$ and from $21 \mathrm{~d}$ to $11 \mathrm{wk}$, whereas among males the number did not significantly change except between 3d and 11wk (Figs. 5A-D and 6). The numbers of $\mathrm{TH}$-ir neurons within the female A8 area seemed to reach to adult level after than in males.

\section{DISCUSSION}

The present study demonstrates the postnatal profiles of age-related changes within the midbrain of the female and 
male $\mathrm{C} 3 \mathrm{H}$ mouse. The rationale for using this strain in this study is, although several other reports have shown the data related to both female and male midbrain DA nuclei by using strains CBA/J [24], BALB/c [19], C57BL/6J [17], and $\mathrm{ddY}$ [35], there is little information about $\mathrm{C} 3 \mathrm{H}$ mice, which are extensively used as experimental animals. The profiles in this study could provide a framework for further investigations of the $\mathrm{C} 3 \mathrm{H}$ mouse midbrain.

The VTA of female mice at $11 \mathrm{wk}$ contained higher levels of TH-ir activity than that of males in the immunohistochemical analysis. While the number of TH-ir cells did not significantly differ between the sexes, there was a female-dominant tendency in $\mathrm{TH}$-ir cell numbers within A10 $(P=0.073)$. However, two other immunostaining groups - that at $3 \mathrm{~d}$ and that at $21 \mathrm{~d}$ - did not show any sex differences. The numbers of TH-ir cells within the A8, A9, and A10 areas peaked at $11 \mathrm{wk}$ in both female and male mice. Some studies have shown decreased TH-ir cell numbers within the midbrain through the ontogenetic period. Tepper et al. (1994) reported a decrease in TH-ir profile counts in the rat SNc between 1 and $14 \mathrm{~d}$, which they interpreted as cell loss [36]. Lieb et al. (1996) also demonstrated a decrease in profile density but an increase in total numbers of TH-ir cells [24]. However, in the present study, we did not detect a decrease in TH-ir cell numbers at any time point. This discrepancy may be attributable to differences in species and strains between our study and the previous ones. Alternatively, the number of TH-ir neurons may decrease at time points other than $3 \mathrm{~d}, 21 \mathrm{~d}$, and $11 \mathrm{wk}$. The numbers of DA neurons in A8, A9, and A10 did not significantly differ between females and males at any time point investigated, supporting the report of Lieb et al. (1996) [24]. However, there was the sex difference in the time period when the DA neuron number significantly increased. This means that the development rate of each DA nucleus within the midbrain differs between sexes. This, in turn, has two implications. First, statistically significant sex differences in the numbers of TH-ir neurons may be found at time points other than $3 \mathrm{~d}, 21 \mathrm{~d}$, and $11 \mathrm{wk}$. Second, sex differences may exist within the midbrain and may reflect the growth rate in DA neuron numbers. The brain of the $11 \mathrm{wk}$ mouse is mature enough to be unsusceptible to sex steroid hormones, whereas the brain at 3 and $21 \mathrm{~d}$ may be affected by gonadal secretions in male mice, which may cause a sex difference in which the growth rate in the number of TH-ir neurons increases.

Many reports have demonstrated functional sex differences within the midbrain DA system affected by sex steroid hormones through the intermediary of AR and/or ERs. Both ER $\alpha$ - and ER $\beta$-ir were expressed in the SNc and VTA of young adult female mice [28]. There are doubleimmunoreactive neurons of AR and TH within the SN, VTA, and RRF of rat [21]; these neurons project to either the amygdala or the nucleus accumbens [10]. ER $\beta$-ir nuclei were found only in neurons, more specifically, within subsets of both DA and nondopaminergic neurons in the dorsal VTA, the PBP, the SNl, and the RRF [9]. Studies suggesting functional sex differences within the midbrain have also been reported. Estrogen acts as a neuroprotective agent differentially between the sexes in neurodegenerative rats administered 6-hydroxydopamine (6-OHDA). Estrogen replacement therapy reduces the lesion size in females but increases it in males [29]. Analysis of DA cell loss within A9 after injury by 6-OHDA administration shows that males are more susceptible to toxicity; female rats have significantly less DA cell loss and respond to 6OHDA with a significantly higher degree of behavioral recovery after the injury [34]. A9 lesions significantly deplete neostriatal dopamine, which is highly correlated with mount latency, mount rate, ejaculation latency, and ejaculation frequency in males [7]. A10 DA neurons, which are sensitive to gonadotropin-releasing hormone, are related to the regulation of lordosis in female rats [33]. These reports strongly suggest that the midbrain DA system includes sex differences underlying the pathogenic mechanism as well as functional sexual dimorphisms. Given that there are sexual dimorphisms in AR and/or ERs, the sensitivity of gonadal secretion will be very different between the sexes. In the present study, the sex differences observed in the maturation rate likely reflect functional sex differences within the midbrain DA system involving cognition, emotion, and behavior.

Chromosome Y genes may also affect the sex-specific development and the functions of the midbrain DA system. One of the genes presumed to cause the male-specific increment pattern of TH-ir neurons is Sry, which is expressed specifically in the brains of adult male mice [13, 26]. The transcript product of the gene is specifically detected in the TH-ir neurons within the SN of the adult male rodent and involves the regulation of mesencephalic TH [12]. An experiment using cell cultures dissociated from midbrains of female and male mice also indicates Sry's involvement in the regulation of DA neurons [8]. The lack of a statistically significant increase in the numbers of TH-ir neurons from 3 to $21 \mathrm{~d}$ in males may reflect individual differences in the complicated mechanisms regulated by male-specific factors, such as neonatal testicular secretion and chromosome Y genes. In females, the absence of malespecific factors might induce 'uniformly' developing patterns of TH-ir neurons that showed statistical significance.

In conclusion, we detected age-related changes within the midbrain of female and male $\mathrm{C} 3 \mathrm{H}$ mice and showed sexspecific development patterns in the numbers of TH-ir neurons there, suggesting that the midbrain DA system may be sexually dimorphic in the maturation process. These differences may reflect the direct regulation of Sry or the multiple effects of both Sry and sex steroid hormones. We have not yet determined clearly which factor forms sexual dimorphism in the midbrain. To elucidate the mechanism underlying female and male development within midbrain DA nuclei, we need to perform further experiments applying gonadectomy and the administration of sex steroid hormones. We also have to determine the meaning of the 
sex-specific increment patterns of TH-ir cells. In light of the fact that there is genetic variation in midbrain DA neuron numbers, the influence levels of hormonal and/or genetic factors may differ according to the inbred strain.

ACKNOWLEDGMENTS. The authors are very grateful to Dr. S. Tsukahara (Division of Environmental Health Sciences, National Institute for Environmental Studies) for his advice and suggestions during this research. This work was supported by Grants-in-Aid for Scientific Research (B) (\#15390510) and for Scientific Research on Priority Areas (\#17052014) from the Ministry of Education, Culture, Sports, Science, and Technology of Japan (to N. Hoshi).

\section{REFERENCES}

1. Baker, H., Joh, T.H. and Reis, D.J. 1980. Genetic control of number of midbrain dopaminergic neurons in inbred strains of mice: Relationship to the size and neuronal density of the striatum. Proc. Nat. Acad. Sci. U.S.A. 77: 4369-4373.

2. Baldereschi, M., Carlo, D., Vanni, P., Maggi, S., Perissinotto, E., Grigoletto, F., Amaducci, L. and Inzitari, D. 2000. Parkinson's disease and parkinsonism in a longitudinal study: Two-fold higher incidence in men. Neurology 55: 1358-1363.

3. Beninger, R.J. 1983. The role of dopamine in locomotor activity and learning, Brain Res. 287: 173-196.

4. Beyer, C., Pilgrim, C. and Reisert, I. 1991. Dopamine content and metabolism in mesencephalic and diencephalic cell cultures: sex differences and effects of sex steroids. $J$. Neurosci. 11: 1325-1333.

5. Bower, J.H., Maraganore, D.M., McDonnell, S.K. and Rocca, W.A. 1999. Incidence and distribution of parkinsonism in Olmsted Country, Minnesota, 1976-1990. Neurology 52: 1214-1220.

6. Bower, J.H., Maraganore, D.M., McDonnell, S.K. and Rocca, W.A. 2000. Influence of strict, intermediate, and broad diagnostic criteria on the age- and sex-specific incidence of Parkinson's disease. Mov. Disord. 15: 819-825.

7. Brackett, N.L., Iuvone, P.M. and Edwards, D.A. 1986. Midbrain lesions, dopamine and male sexual behavior. Behav. Brain Res. 20: 231-240.

8. Carruth, L.L., Reisert, I. and Arnold, A.P. 2002. Sex chromosome genes directly affect brain sexual differentiation. Nat. Neurosci. 5: 933-934.

9. Creutz, L.M. and Kritzer, M.F. 2002. Estrogen receptor-beta immunoreactivity in the midbrain of adult rats: regional, subregional, and cellular localization in the A10, A9, and A8 dopamine cell groups. J. Comp. Neurol. 446: 288-300.

10. Creutz, L.M. and Kritzer, M.F. 2004. Mesostriatal and mesolimbic projections of midbrain neurons immunoreactive for estrogen receptor beta or androgen receptors in rats. $J$. Comp. Neurol. 476: 348-362.

11. Dahlstroem, A. and Fuxe, K. 1964. Evidece for the existence of monoamine-conteining neurons in the central nervous system. I. Demonstration of monoamines in the cell bodies of brain stem neurons. Acta. Physiol. Scand. 62: (Suppl. 232): 1-55.

12. Dewing, P., Chiang, C.W.K., Sinchak, K., Sim, H., Fernagut, P.O., Kelly. S., Chesselet, M.F., Micevych, P.E., Albrecht, K.H., Harley, V.R. and Vilain, E. 2006. Direct regulation of adult brain function by the male-specific factor SRY. Curr. Biol. 16: 415-420.
13. Dewing, P., Shi, T., Horvath, S. and Vilain, E. 2003. Sexually dimorphic gene expression in mouse brain precedes gonadal differentiation. Brain Res. Mol. Brain Res. 118: 82-90.

14. Diamond, S.G., Markham, C.H., Hoehn, M.M., McDowell,. F.H. and Muenter, M.D. 1990. An examination of male-female differences in progression and mortality of Parkinson's disease. Neurology 40: 763-766.

15. Faraone, S.V. and Biedermann, J. 1998. Neurobiology of attention-deficit hyperactivity disorder. Biol. Psychiatry 44: 951-958.

16. Gorski, R.A., Gordon, J.H., Shryne, J.E. and Southam, A.M. 1978. Evidence for a morphological sex difference within the medial preoptic area of the rat brain. Brain Res. 148: 333-346.

17. Hamre, K., Tharp, R., Poon, K., Xiong, X. and Smeyne, R.J. 1999. Differential strain susceptibility following 1-methyl-4phenyl-1,2,3,6-tetrahydropyridine (MPTP) administration acts in an autosomal dominant fashion: quantitative analysis in seven strains of Mus musculus. Brain Res. 828: 91-103.

18. Hornykiewicz, O. 1979. Brain dopamine in Parkinson's Disease and other neurological disturbances. pp 643-654. In: The Neurobiology of dopamine (Horn, A.S., Dorf, J. and Westerink, B.H.C. eds.), Academic Press, London.

19. Ivanova, T. and Beyer, C. 2003. Estrogen regulates tyrosine hydroxylase expression in the neonate mouse midbrain. $J$. Neurobiol. 54: 638-647.

20. Koob, G.F. 1999. The role of the striatopallidal and extended amygdala systems in drug addiction. Ann. New York Acad. Sci. 877: 445-460.

21. Kritzer, M. 1997. Selective colocalization of immunoreactivity for intracellular gonadal hormone receptors and tyrosine hydroxylase in the ventral tegmental area, substantia nigra, and retrorubral fields in the rat. J. Comp. Neurol. 379: 247-260.

22. Kuhnemann, S., Brouwn, T.J., Hochberg, R.B. and MacLusky, N.J. 1994. Sex differences in the development of estrogen receptors in the rat brain. Horm. Behav. 28: 483-491.

23. Lahr, G., Maxson, S.C., Mayer, A., Just, W., Pilgrim, C. and Reisert, I. 1995. Transcription of the Y chromosomal gene, Sry, in adult mouse brain. Brain Res. Mol. Brain. Res. 33: 179182.

24. Lieb, K., Andersen, C., Lazarov, N., Zienecker, R., Urban, I., Reisert, I. and Pilgrim, C. 1996. Pre- and postnatal development of dopaminergic neuron numbers in the male and female mouse midbrain. Brain Res. Dev. Brain Res. 94: 37-43.

25. MacLusky, N.J. and Naftolin, F. 1981. Sexual differentiation of the central nervous system. Science 211: 1294-1303.

26. Mayer, A., Mosier, G., Just, W., Pilgrim, C. and Reisert, I. 2000. Developmental profile of Sry transcripts in mouse brain. Neurogenetics 3: 25-30.

27. Meltzer, H.Y. and Stahl, S.M. 1976. The dopamine hypothesis of schizophrenia: A review. Schizophr. Bull. 2: 19-76.

28. Mitra, S.W., Hoskin, E., Yudkovitz, J., Pear, L., Wilkinson, H.A., Hayashi, S., Pfaff, D.W., Ogawa, S., Rohrer, S.P., Schaeffer, J.M., McEwen, B.S. and Alves, S.E. 2003. Immunolocalization of estrogen receptor $\beta$ in the mouse brain: Comparison with estrogen receptor $\alpha$. Endocrinology 144: 2055-2067.

29. Murray, H.E., Pillai, A.V., Mcarthur, S.R., Razvi, N., Datla, K.P., Dexter, D.T. and Gillies, G.E. 2003. Dose- and sexdependent effects of the neurotoxin 6-hydroxydopamine on the nigrostriatal dopaminergic pathway of adult rats: Differential actions of estrogen in males and females. Neuroscience 116: 213-222. 
30. Nemeroff, C.B. and Bisette, G. 1988. Neuropeptides, dopamine, and schizophrenia. Ann. New York Acad. Sci. 537: 273-291.

31. Paxinos, G. and Franklin, K.B.J. 2001. The Mouse Brain in Stereotaxic Coordinates, second ed., Academic Press, Hong Kong.

32. Simerly, R.B., Zee, M.C., Pendleton, J.W., Lubahn, D.B. and Korach, K.S. 1997. Estrogen receptor-dependent sexual differentiation of dopaminergic neurons in the preoptic region of the mouse. Proc. Natl. Acad. Sci. U.S.A. 94: 14077-14082.

33. Sirinathsinghji, D.J., Whittington, P.E. and Audsley, A.R. 1986. Regulation of mating behavior in the female rat by gonadotropin-releasing hormone in the ventral tegmental area: Effects of selective destruction of the A10 dopamine neurons. Brain Res. 374: 167-173.

34. Tamas, A., Lubics, A., Szalontay, L., Lengvari, I. and Reglodi, D. 2005. Age and gender differences in behavioral and morphological outcome after 6-hydroxydopamine-induced lesion of the substantia nigra in rats. Behav. Brain Res. 158:
221-229.

35. Tando, S., Itoh, K., Yaoi, T., Ikeda, J., Fujiwara, Y. and Fushiki, S. 2007. Effects of pre- and neonatal exposure to bisphenol A on murine brain development. Brain Dev. 29: 352-356.

36. Tepper, J.M., Damlama, M. and Trent, F. 1994. Postnatal changes in the distribution and morphology of rat substantia nigra dopaminergic neurons. Neuroscience 60: 469-477.

37. Vadasz, C., Baker, H., Fink, S.J. and Reis, D.J. 1985. Genetic effects and sexual dimorphism in tyrosine hydroxylase activity in two mouse strains and their reciprocal F1 hybrids. $J$. Neurogenet. 2: 219-230.

38. Yamaguchi, S. and Kobayashi, S. 1998. Contributions of the dopaminergic system to voluntary and automatic orienting of visuospatial attention. J. Neurosci. 18: 1869-1878.

39. Zaborszky, L. and Vadasz, C. 2001. The midbrain dopaminergic system: Anatomy and genetic variation in dopamine neuron number of inbred mouse strains. Behavior Genetics 31: 47-59. 